\title{
Comparison of Fixed and Variable Pitch Actuators for Agile Quadrotors
}

\author{
Mark Cutler* N. Kemal Ure ${ }^{\dagger} \quad$ Bernard Michini $^{\dagger} \quad$ Jonathan P. How ${ }^{\ddagger}$
}

This paper presents the design, analysis and experimental testing of a variablepitch quadrotor. A custom in-lab built quadrotor with on-board attitude stabilization is developed and tested. An analysis of the dynamic differences in thrust output between a fixed-pitch and variable-pitch propeller is given and validated with simulation and experimental results. It is shown that variable-pitch actuation has significant advantages over the conventional fixed-pitch configuration, including increased thrust rate of change, decreased control saturation, and the ability to quickly and efficiently reverse thrust. These advantages result in improved quadrotor tracking of linear and angular acceleration command inputs in both simulation and hardware testing. The benefits should enable more aggressive and aerobatic flying with the variable-pitch quadrotor than with standard fixed-pitch actuation, while retaining much of the mechanical simplicity and robustness of the fixed-pitch quadrotor.

\section{Nomenclature}

$\begin{array}{llll}\alpha & \text { Propeller pitch angle } & \omega & \text { Motor speed } \\ \rho & \text { Air density } & b_{D 1}, \ldots b_{D 3} & \text { Estimated drag coefficients } \\ b_{L} & \text { Estimated lift coefficient } & c & \text { Propeller cord } \\ R & \text { Motor internal resistance } & L & \text { Motor inductance } \\ C_{D 0} & \text { Parasitic drag coefficient } & C_{D i} & \text { Lift-induced drag coefficient } \\ C_{L \alpha} & \text { Lift coefficient } & C_{L 0} & \text { Lift coefficient at zero pitch } \\ C_{L m i n} & \text { Minimum lift coefficient } & C_{L m a x} & \text { Maximum lift coefficient } \\ e & \text { Motor back EMF } & i & \text { Motor current } \\ i_{0} & \text { Motor no-load current } & I & \text { Motor and propeller inertia } \\ K_{V} & \text { Motor voltage constant } & K_{Q} & \text { Motor torque constant } \\ R E_{e x p} & \text { Reynolds number adjusting factor } & R_{p} & \text { Propeller radius } \\ R E_{r e f} & \text { Reynolds number for drag calculations } & v & \text { Motor voltage } \\ v_{L} & \text { Motor voltage from inductance } & T_{L} & \text { Load torque } \\ v_{R} & \text { Motor voltage from resistance } & T_{M} & \text { Motor torque }\end{array}$

\section{Introduction}

Quadrotor helicopters are widely used as experimental platforms due to their mechanical simplicity and inherent robustness. In traditional fixed-pitch quadrotors, stability and flight control

${ }^{*}$ Research assistant in the Aerospace Controls Lab at MIT cutlerm@mit.edu

${ }^{\dagger}$ Ph.D. candidate in the Aerospace Controls Lab at MIT ure, bmich\}@mit.edu

${ }^{\ddagger}$ Richard C. Maclaurin Professor of Aeronautics and Astronautics, MIT. Associate Fellow AIAA. jhow@mit.edu 
are achieved by changing the speed of each of the four motors. ${ }^{1-4}$ While differential RPM control is sufficient for most flight regimes and can result in agile and aggressive flights, ${ }^{5-8}$ it places fundamental constraints on the vehicle as the flight envelope expands. In particular, control bandwidth is limited by the rotational inertia of the motors and fixed-pitch quadrotors are unable to efficiently achieve reverse thrust. These constraints limit the aerobatic maneuvers a quadrotor can perform, therefore limiting the future applicability of quadrotors in agile intensive missions. These limitations in fixed-pitch quadrotors are overcome with the addition of variable-pitch propellers. While variable-pitch propellers add some complexity to an otherwise simple and relatively robust quadrotor, the advantages of increased controller bandwidth and reverse thrust capabilities could justify the design when aggressive and agile flight is required.

Controller bandwidth can be a significant problem for quadrotors, and becomes an issue for quadrotor stability as the size of the quadrotor increases. ${ }^{9}$ Larger quadrotors require larger motors which, in turn, have larger inertias and cannot be controlled as quickly as smaller motors. Eventually, as the size increases enough, the quadrotor can no longer be stabilized through RPM control alone because the torque required to change the rotational velocity of the motor quickly exceeds the capacity of the motor. Thus, variable-pitch blades may be necessary for larger quadrotors merely for stabilization purposes.

For small quadrotors (less than about $2 \mathrm{Kg}$ ), however, motor inertias are small and adequate control bandwidth can be achieved with fixed-pitch propellers. Fixed-pitch quadrotors have demonstrated relatively aggressive maneuvers. ${ }^{6,7,10,11}$ However, traditional pod-and-boom autonomous helicopters have demonstrated agile maneuvers outside of the flight regime of small fixed-pitch quadrotors. ${ }^{12,13}$ The proposed variable-pitch quadrotor attempts to bridge the gap between simple, robust, fixed-pitch quadrotors and aggressive, complicated, and fragile helicopters. The variablepitch quadrotor presents a simple, yet agile platform for robust, aggressive maneuvering.

Several variable-pitch quadrotors have already been built. ${ }^{14,15}$ One quadrotor has even demonstrated autonomous upright and inverted flight and flips. ${ }^{16}$ Some large-scale rotorcraft such as Convertawing's Model "A" quadrotor in 1956 achieved flight with variable-pitch propellers. The proposed variable-pitch quadrotor, however, is among the first documented small variable-pitch quadrotors to demonstrate stable upright and inverted hovering and waypoint tracking, in addition to flipping from upright to inverted flight. ${ }^{17}$ The addition of variable-pitch propellers to a quadrotor increases the vehicle's potential agility by increasing the thrust rate of change, increasing the potential deceleration of the vehicle to greater than gravity, and allowing for inverted flight.

This paper is organized as follows. Section II gives a brief overview of the hardware used on the variable-pitch quadrotor. Section III details the aerodynamic effects of adding a variable-pitch propeller to a quadrotor. Simulations of the variable-pitch quadrotor compared with a fixed-pitch quadrotor are presented in Section IV followed by experimental results in Section V. Finally, Section VI summarizes the results and advantages of a variable-pitch quadrotor.

\section{Hardware}

The variable pitch quadrotor proposed here uses four 10-gram MKS digital high-speed servos to control the pitch angle of the blade via a control rod that runs through a hollow motor shaft. Brushless motors are driven by open-source Mikrokopter electronic speed controllers. Inertial measurement, filtering, and high-rate attitude stabilization is achieved using a 12 gram customizable autopilot developed by Unmanned Innovation. ${ }^{18}$ The autopilot connects to a quadrotor specific breakout board that contains an xBee radio modem, power regulator, and connectors. The electronics, motors, and propellers are all mounted on a custom carbon fiber frame.

Figure 1 shows the quadrotor with the variable-pitch propellers. The servos and all of the variable-pitch components combined add only about 90 grams to the overall weight of the quadro- 


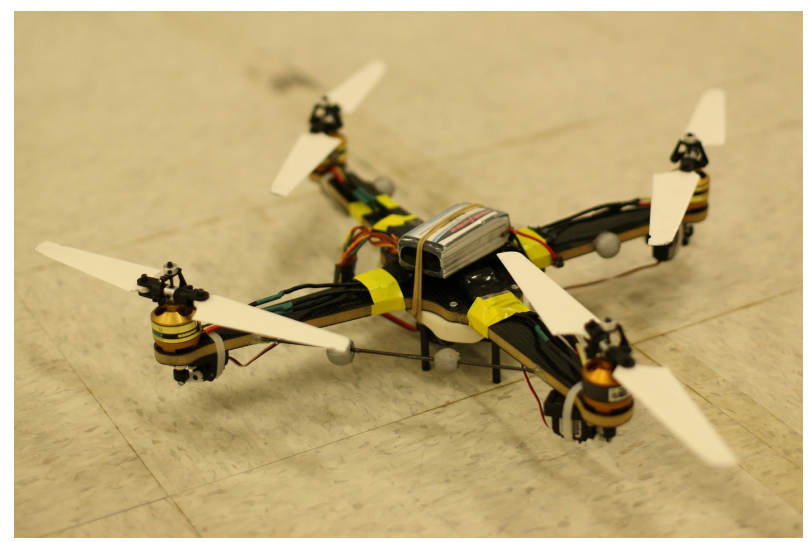

Figure 1. Variable-pitch quadrotor designed at the Aerospace Controls Lab. The servos that actuate the variable-pitch propellers are visible under each of the motors. The quadrotor measures $0.35 \mathrm{~m}$ across.

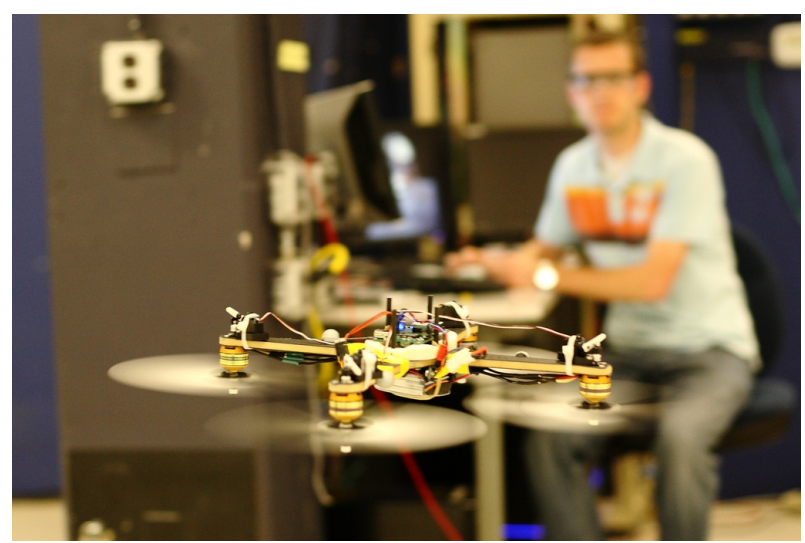

Figure 2. The quadrotor during inverted waypoint tracking. It has successfully flown upright, inverted, and autonomously flipped between the two.

tor, where the motors alone weigh nearly 200 grams. As seen, the variable-pitch propellers are mechanically simple, preserving the robustness of the original concept of the quadrotor. Swashplates, needed on true helicopters, are much more mechanically complex and prone to failure than simple variable-pitch actuation. Thus the variable-pitch quadrotor represents a relatively small increase in both hardware complexity and added weight.

\section{Thrust Actuation}

With fixed-pitch propellers the thrust produced by the propeller is constant given a constant motor rotational rate (assuming the quadrotor is near hover). Therefore, the only way to change the thrust produced by the propeller is by changing the voltage to the motor and therefore varying the rotational rate of the propeller.

Adding variable-pitch propellers to the quadrotor platform results in an additional degree of freedom for varying the thrust produced by each motor-propeller combination. With variable-pitch propellers, thrust can be changed by either changing the blade pitch or by changing the rotational rate of the motors. These two actuators, to a large extent, overlap. For instance, with variablepitch propellers a quadrotor can hover using high RPM and low blade pitch, or using low RPM and high blade pitch, or any combination in between.

The output of the quadrotor attitude control loop is assumed to be a desired thrust. This section addresses the benefits of adding variable-pitch control to a quadrotor. The allocation problem of which actuator to use, pitch or motor speed, is also addressed.

\section{A. Propeller Model}

Analytical analysis of the variable-pitch actuation requires a detailed study of the propeller aerodynamics. The propellers used on the variable-pitch quadrotor are symmetric, tapered, nine inch diameter blades. To determine the lift and drag coefficients of the propellers to use in the analysis, an airfoil, operating Reynolds number, and Mach number must be selected. The maximum thickness to chord ratio is 0.899 , and so a NACA 0009 airfoil is chosen to model the propeller airfoil. Assuming the blades are rotating at 8000 RPM, the Mach number near the end of the blades is about 0.25 . This gives an operating Reynolds number of around 100,000. 
Table 1. Propeller Aerodynamic Coefficients

\begin{tabular}{cccccccccc}
\hline$C_{L 0}$ & $C_{L a}$ & $C_{L m i n}$ & $C_{L \max }$ & $C_{D 0}$ & $C_{D 2 u}$ & $C_{D 2 l}$ & $C_{L} C_{D 0}$ & $R E_{r e f}$ & $R E_{\text {exp }}$ \\
0.0 & 2.87 & 0.0 & 0.7 & 0.01 & 0.0408 & 0.0408 & 0.0 & 100000 & -0.5 \\
\hline
\end{tabular}

Table 2. Motor Coefficients

\begin{tabular}{ccc}
\hline$K_{V}(\mathrm{rad} / \mathrm{s} /$ volts $)$ & $R$ (Ohms) & $i_{0}$ (Amps) \\
115.2 & 0.26 & 0.35 \\
\hline
\end{tabular}

Using XFOIL, ${ }^{19}$ aerodynamic coefficients for the propeller blades were determined and for reference are displayed in Table 1.

\section{B. Motor Model}

The variable-pitch quadrotor uses electric DC brushless hobby motors. Electric DC motors are well modeled by a circuit containing a resistor, inductor, and voltage generator in series. ${ }^{20}$ Kirchhoff's law applied to the equivalent motor circuit yields

$$
v=v_{R}+v_{L}+e
$$

which can be rewritten as

$$
v=R i+L \frac{\partial i}{\partial t}+\frac{\omega}{K_{V}}
$$

where $R$ is the motor internal resistance, $L$ is the inductance, $\omega$ is the rotational rate of the motor, and $K_{V}$ is the voltage constant of the motor, expressed in rad/s/volt. Also, the motor torque, $T_{M}$ can be modeled as being proportional to the difference between the current, $i$, and the no-load current, $i_{0}$, through the torque constant, $K_{Q}$, expressed in Amp/Nm.

$$
T_{M}=\frac{\left(i-i_{0}\right)}{K_{Q}}
$$

The motor dynamics are modeled as a simple first order differential equation in Eq 4 where $\dot{\omega}$ is driven by the motor torque and the load torque, $T_{L}$. The inertia, $I$, includes the motor and the propeller, $T_{M}$ comes from the voltage generator, and $T_{L}$ results from the propeller drag.

$$
I \dot{\omega}=T_{M}-T_{L}
$$

Typically, the inductance of small, brushless hobby motors is small when compared to the physical response of the system and so can be ignored. ${ }^{20}$ Substituting Eq. 2 and 3 into Eq. 4 and neglecting the inductance term yields the following differential equation for the motor speed, $\omega$

$$
I \dot{\omega}=\left[\left(v-\frac{\omega}{K_{V}}\right) \frac{1}{R}-i_{0}\right] \frac{1}{K_{Q}}-T_{L}
$$

Table 2 shows the motor coefficients for the AXI 2208/34 brushless motors used on the variablepitch quadrotor. 


\section{Nonlinear Motor-Propeller Model}

$\mathrm{QPROP}^{21}$ is used to determine the steady state values of the load torque due to the drag, $T_{L}$, and the lift, $L$, generated by the propellers as functions of $\omega$ and the pitch angle, $\alpha$. Fig. 3 and 4 show the output of QPROP. In each plot, lines of constant thrust $(\mathrm{N})$ are denoted by red numbers and lines of constant motor speed (RPM) are denoted by blue numbers. The grey areas demonstrate operating regimes that should be avoided due to motor vibrations and propeller stall. In Fig. 3, the approximate hover thrust required by each of the four motors is denoted by the green line-indicating the many combinations of pitch and voltage settings that could be used to hover. The plot also shows how thrust can be increased from hover by either increasing motor voltage, increasing pitch, or increasing both. In Fig. 4 the y axis shows power drawn by the motor.

Assuming the quadrotor is near hover, the lift and drag generated by the propeller can be modeled by ${ }^{22}$

$$
\begin{aligned}
L & =\rho c R_{p}^{3} \omega^{2} C_{L \alpha} \frac{\alpha}{3} \\
T_{L} & =\rho c R_{p}^{4} \omega^{2}\left(\frac{C_{D 0}+C_{D i} \alpha^{2}}{4}-\frac{C_{L \alpha} \alpha \omega}{3 R_{p}}\right)
\end{aligned}
$$

These equations show that the lift and drag produced by the motor-propeller combination is affected by the motor speed, $\omega$, and the propeller pitch angle, $\alpha$. The other terms in the two equations are constants. Using least-squares regression, the data generated by QPROP is used to estimate these constants. The numerical values of those constants are shown in Table 3.

$$
\begin{aligned}
L & =b_{L} \omega^{2} \alpha \\
T_{L} & =b_{D_{1}} \omega^{2}+b_{D_{2}} \omega^{2} \alpha^{2}+b_{D_{3}} \omega \alpha
\end{aligned}
$$

Substituting Eq. 9 into Eq. 5 results in the following nonlinear differential equation for $\dot{\omega}$

$$
I \dot{\omega}=\left[\left(v-\frac{\omega}{K_{V}}\right) \frac{1}{R}-i_{0}\right] \frac{1}{K_{Q}}-b_{D_{1}} \omega^{2}-b_{D_{2}} \omega^{2} \alpha^{2}-b_{D_{3}} \omega \alpha
$$

Table 3. Estimated Lift and Drag Coefficients

\begin{tabular}{cccc}
\hline$b_{L}$ & $b_{D 1}$ & $b_{D 2}$ & $b_{D 3}$ \\
$3.88 \mathrm{e}-07$ & $9.96 \mathrm{e}-09$ & $2.46 \mathrm{e}-10$ & $4.33 \mathrm{e}-07$ \\
\hline
\end{tabular}

\section{Linearized Motor-Propeller Model}

To develop further insights into the dynamics, the nonlinear equations can be linearized about the hover conditions $\omega_{0}$ and $\alpha_{0}$ The resulting state-space system is

$$
\begin{aligned}
& \Delta \dot{\omega}=\left[-\frac{1}{R K_{V} K_{Q} I}-\frac{2 b_{D_{1}} \omega_{0}}{I}-\frac{2 b_{D_{2}} \omega_{0} \alpha_{0}^{2}}{I}-\frac{b_{D_{3}} \alpha_{0}}{I}\right] \Delta \omega+\left[\frac{1}{R K_{Q} I} \frac{-2 b_{D_{2}} \omega_{0}^{2} \alpha_{0}}{I}-\frac{-b_{D_{3}} \omega_{0}}{I}\right]\left[\begin{array}{c}
\Delta v \\
\Delta \alpha
\end{array}\right] \\
& \Delta L=\left[2 b_{L} \omega_{0} \alpha_{0}\right] \Delta \omega \quad+\left[\begin{array}{ll}
0 & b_{L} \omega_{0}^{2}
\end{array}\right]\left[\begin{array}{c}
\Delta v \\
\Delta \alpha
\end{array}\right]
\end{aligned}
$$

The output of this system of equations is the change in lift $(\Delta L)$ and the two inputs are the change in voltage $(\Delta v)$ and change in pitch $(\Delta \alpha)$. 
These linearized equations encapsulate the fundamental difference between variable-pitch and fixed-pitch actuators. With a fixed-pitch propeller, thrust is generated by increasing the motor voltage. This motor voltage must first increase the motor speed, fundamentally limiting the rate of lift increase by the dynamics of the motor. However, with a variable-pitch actuator, there is a significant non-zero direct feed-through term in the lift equation. Any change in the pitch of the blades directly affects lift, bypassing the motor dynamics. Of course, increasing the pitch of the propellers negatively impacts the speed of the motor and therefore negatively impacts the lift produced, but that decrease in lift is first filtered through the motor dynamics. As long as the mechanism for actuating pitch is fast when compared to the response of the motor, varying the pitch of the blades results in faster changes in thrust than varying the motor voltage.

Nonlinear simulation results from the data generated by QPROP for lift and motor speed responses to steps in voltage and pitch are shown in Fig. 5. The direct feed-through from pitch to lift is apparent as the lift instantaneously increases when the step in pitch is commanded. The lift then decreases as the increased torque on the motor slows the motor down to the new steady state value. Graphically, these two different actuators are shown in Fig. 3. The blue line indicates a step in voltage from hover while the red line represents the corresponding step in pitch from the same initial conditions. The diagonal green line shows a similar change in thrust by using both voltage and pitch inputs. This thrust change lies roughly parallel with the lines of constant motor speed, indicating that the motor speed has not changed, or that the motor dynamics have essentially been cancelled during the thrust change. The canceling of the slow motor dynamics during thrust change illustrates an advantage of using both voltage and pitch control in combination.

\section{E. Hardware Effects}

The linearized equations of motion in Eq. 11 make a number of simplifying assumptions. In addition to the error introduced through linearization, the model assumes both voltage and pitch can be changed instantaneously and continuously. In reality, the voltage to the motor first passes through the dynamics of the electronic speed controller (ESC). Bench tests suggest that the dynamics of the ESC can be approximated as a rate limiter of 70 volts/sec. Also, on the actual quadrotor, the propeller pitch is actuated by a digital servo. The servo dynamics are approximately modeled by a rate limit and a small lag. No load specifications on the servos indicate a rate limit of about 900 degrees/sec. Bench test data indicates that the loaded response is roughly $70 \%$ of that value.

Actuator update rate can also affect the time responses of the two actuators. Bench testing of fixed-pitch quadrotors has demonstrated that increasing the update rate of the command signal to the ESC from $200 \mathrm{~Hz}$ to $500 \mathrm{~Hz}$ greatly improves the visible hover performance of the vehicle. The ESC's on the variable-pitch quadrotor are controlled over an $\mathrm{I}^{2} \mathrm{C}$ bus and accept updates at up to $1000 \mathrm{~Hz}$. The servos, however, accept PWM commands at a maximum of $333 \mathrm{~Hz}$. Assuming the attitude loop is running at $1000 \mathrm{~Hz}$ or faster, the ESCs can accept commands three times as fast as the servos.

Figures 6(a) and 6(b) show the same step responses from Fig. 5 with the effects of the rate limiters, the servo lag and the delay. The delay is modeled as a zero-order hold (ZOH). Digital control theory ${ }^{23}$ indicates that a $\mathrm{ZOH}$ is well modeled as a delay of one half the update rate of the actuator. To analyze a worst-case scenario, the $\mathrm{ZOH}$ from the update rate is modeled with a delay equal to the update rate of the actuator. Fig. 6(c) shows effects of this worst-case analysis of the update rates. The thrust initially increases faster when the voltage is changed, but the servo quickly overtakes it. The initially faster change in thrust with voltage control may improve flight during maneuvers such as hovering where small, fast thrust changes are required to fly smoothly. 


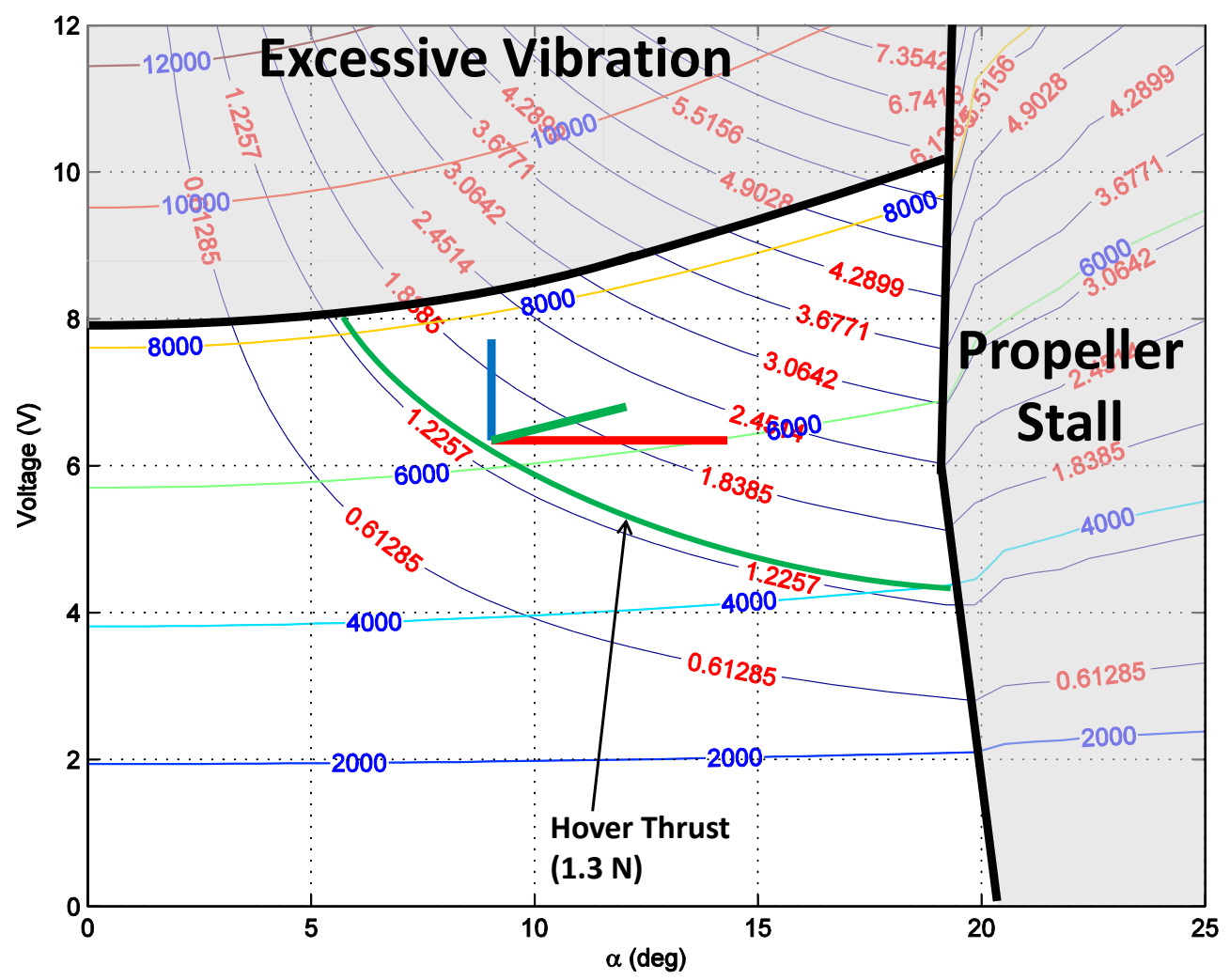

Figure 3. Thrust (N), displayed with red numbers, and motor speed (RPM), displayed in blue numbers, as a function of voltage and pitch.

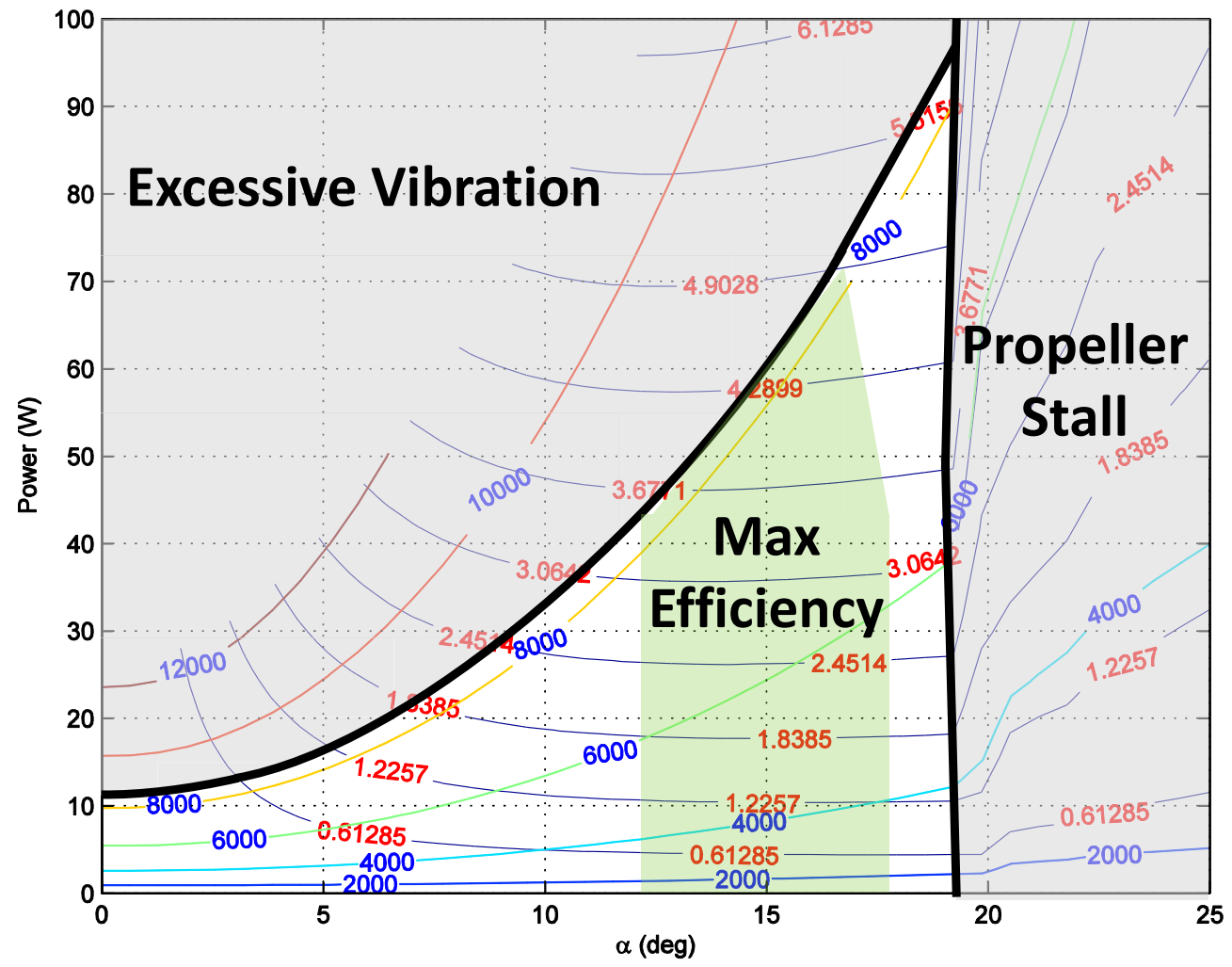

Figure 4. Thrust (N), displayed with red numbers, and motor speed (RPM), displayed in blue numbers, as a function of power and pitch. 


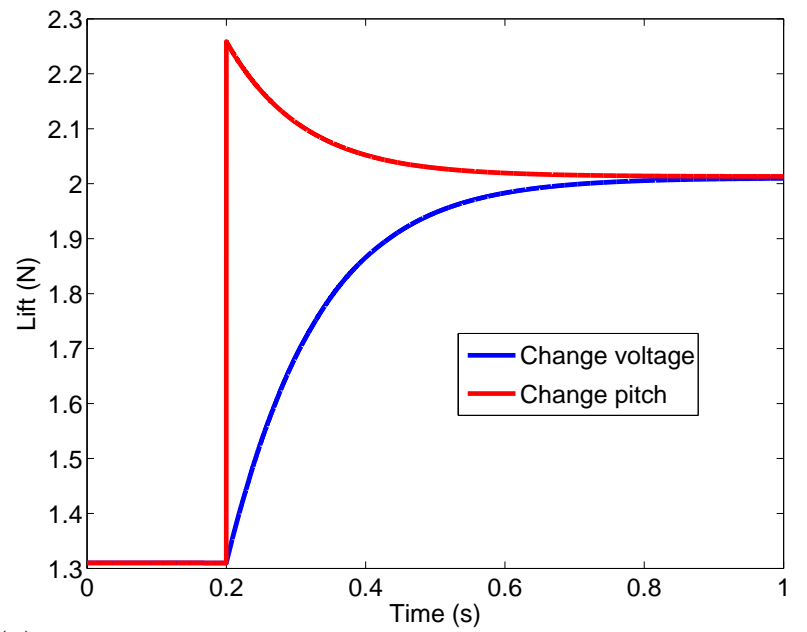

(a) Simulated thrust response to steps in voltage and pitch.

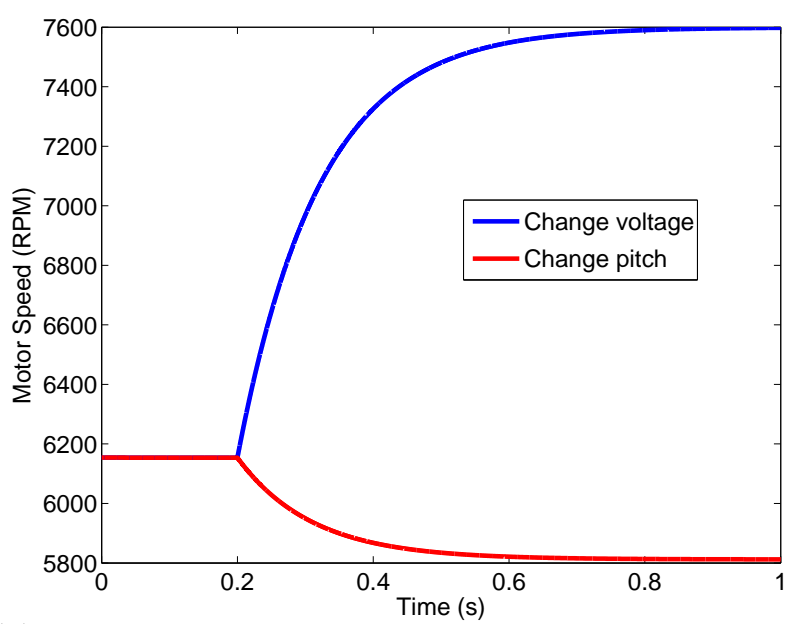

(b) Simulated motor speed response to steps in voltage and pitch.

Figure 5. Linear step responses - step occurs at 0.2 seconds.

\section{F. Control Allocation}

Deciding which thrust actuation mechanism or combination of the two to use depends on what the control objectives are. For instance, if you are concerned about minimizing power consumption, Fig. 4 suggests keeping the blade pitch between 12-18 degrees when hovering - the constant thrust lines have minimum power in this range. However, if you are instead concerned about the ability to quickly increase the thrust of the motors, hovering with a lower pitch and higher RPM allows you to increase the pitch more before stalling the propellers. In other words, the motor-propeller combination contains more energy at hover that can be quickly converted into thrust by increasing propeller pitch.

Agile and aggressive flight ${ }^{5}$ requires quick changes in thrust. It follows that for agile flight the kinetic energy stored in the motor should be maximized. As indicated in Fig. 3, tests reveal that vibration from the motors becomes excessive for the frame and autopilot around 8000 RPM, placing an upper limit on the kinetic energy that can be stored in the motor. Keeping the energy high under varying pitch values implies that the power to the motor must be adjusted to compensate for the varying propeller load.

One strategy for agile flight is to always keep the motor speed at its upper limit. Solving Eq. 5 for $\Delta v$ when $\dot{\omega}=0$ yields

$$
\Delta v=R K_{Q} \Delta T_{L},
$$

the required change in voltage as the pitch is changed to keep the RPM constant. Adding power to the motor as the pitch is increased to keep the motor speed constant is effectively canceling the effects of the motor dynamics. Figures 6(a) and 6(b) demonstrate that this strategy increases the thrust quickly while avoiding a RPM and thrust decrease after the increase in pitch.

\section{Simulation}

In Section III, the dynamic relationships between thrust, pitch, and motor speed were developed. In this section, two non-linear simulations of the quadrotor using the data from QPROP in Section III are used to show the extent to which variable-pitch propellers increase the flight performance of the vehicle.

In the first simulation, only vertical flight is considered. The quadrotor is commanded to follow 


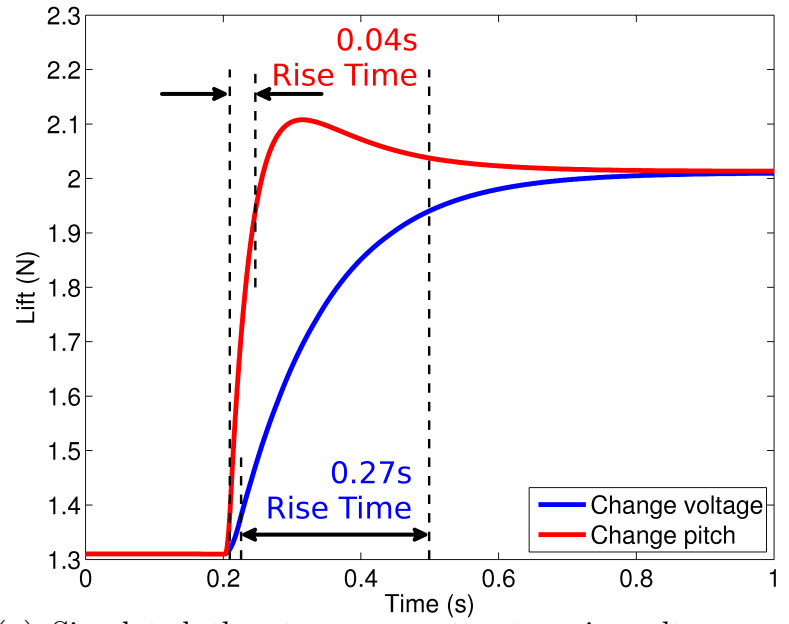

(a) Simulated thrust response to steps in voltage and pitch.

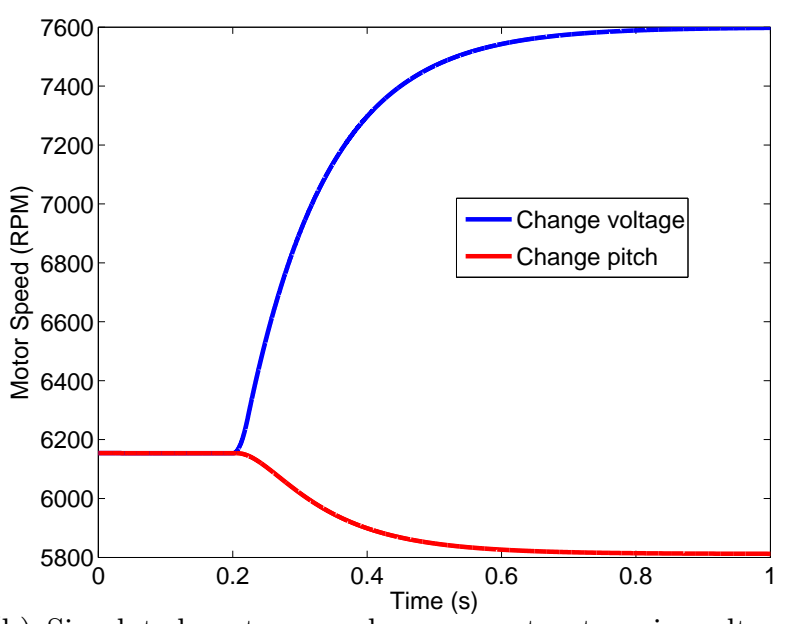

(b) Simulated motor speed response to steps in voltage and pitch.

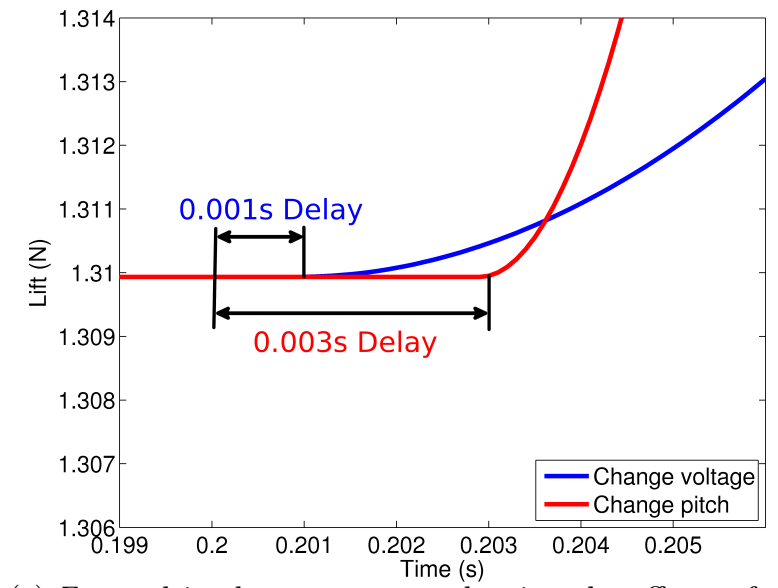

(c) Zoomed in thrust response showing the effects of the actuator digital update rates.

Figure 6. Simulated step responses with rate limit, servo lag, and delay effects.

a sequence of constant vertical velocity commands. For several different velocity commands, the performance of the vehicle is considered using both fixed-pitch (motor voltage commands) and variable-pitch control. The quadrotor is simulated in closed-loop control using a proportionalderivative $(\mathrm{PD})$ controller with position and velocity feedback. Since the two actuators generate different thrust values for the same input command, the PD gains for both actuators were selected to ensure the dominate linearized closed-loop poles are in the same location for both cases. Thus the two cases can be compared with each other despite being in closed-loop control.

Fig. 7 shows the time response of the simulated vertical tracking when the commanded vertical velocity is $0.5 \mathrm{~m} / \mathrm{s}$. Note that the closed-loop position and velocity responses are roughly the same for the two actuators since the dominant closed-loop poles are in the same location. Interestingly, though, the fixed-pitch controller commands a much higher thrust input (thrust and acceleration are directly proportional) to get the same response since the actuator is slower than the variablepitch case. When under variable-pitch control, though, the thrust reference commands are lower because the actuator is able to keep the control error significantly smaller.

Fig. 8 demonstrates that as the commanded vertical velocity is increased to $3.5 \mathrm{~m} / \mathrm{s}$, the two cases differ significantly both in reference command and in closed-loop time response. With larger velocity errors, the fixed-pitch controller's need to create large reference commands is even more 
pronounced than in Fig. 7. Also, another of the fundamental differences between the two actuators is prominent in this plot. When the propeller pitch is fixed, the quadrotor's ability to decelerate is limited by gravity since the motor cannot efficiently reverse its direction mid flight; however, when the pitch is controlled, large decelerations are realized due to the propellers' ability to quickly transition from positive to negative thrust. The acceleration plot in Fig. 8(a) shows that even though the fixed-pitch controller requests accelerations below gravity, the actuator is saturated and cannot follow the reference command, resulting in a large overshoot in the position response.

The vertical tracking performance of the vehicle when in fixed-pitch and variable-pitch modes using controllers with the same dominant closed-loop poles is summarized in Fig. 9. Each dot on the graph shows the sum of the absolute value of the position error at each time-step during the simulation. As expected, at low reference velocities the controllers have roughly the same error since the controllers perform similarly. However, the errors start drastically diverging at around $2 \mathrm{~m} / \mathrm{s}$ commanded velocity, the speed at which the fixed-pitch controller's deceleration starts saturating.

The second simulation considers only the roll axis of the quadrotor. The vehicle is commanded to follow a sequence of constant angular acceleration commands. Constant acceleration is commanded instead of constant velocity as in the vertical simulation because the smoother command shaping greatly improves quadrotor response with the lightly damped roll axis. As in the vertical simulation, the controller used is a PD controller with roll and roll rate feedback and the controller gains are selected so that the two cases have the same dominant closed-loop pole locations. Fig. 10 shows the roll, roll rate, and roll acceleration time responses for the two different actuators when the commanded roll acceleration is $2500 \mathrm{deg} / \mathrm{s}^{2}$. Again, the fixed-pitch controller requests much larger reference thrust commands than the variable-pitch controller in order to get similar roll time responses.

The accumulated roll and roll rate tracking errors as a function of commanded roll rate is displayed in Fig. 11. As in the vertical flight simulation, the fixed-pitch and variable-pitch actuators perform similarly when the commanded angular acceleration is low. However, as the commanded acceleration, or aggressiveness, increases, the fixed-pitch propellers begin to saturate while the variable-pitch propellers do not. Theoretically, the variable-pitch propellers can produce at least twice the torque to the vehicle when rolling as the fixed-pitch propellers because of the ability to reverse the direction of thrust on one of the motors. Thus, the fixed-pitch propellers begin to saturate as one motor produces nearly zero thrust and the other motor produces maximum thrust. This saturation is clearly evident in the error plot as the fixed-pitch error diverges from the variable-pitch error.

Another benefit of variable-pitch propellers when rotating about an axis lies in their ability to quickly track a reference command. This is more clearly shown in the linearized root locus plots of the closed-loop roll axis. For both actuators the plant consists of three poles, two of which are at the origin for the roll axis. The variable-pitch actuator adds an additional zero near the motor pole coming from the non-zero pitch feed through term in Eq. 11. Fig. 12 shows that although the two controllers have placed the closed-loop poles in roughly the same location, the gain on the variablepitch controller in Fig. 12(b) can still be increased significantly to improve the time response of the system. However, it is clear in Fig. 12(a) that moving the compensator pole towards the origin to increase the speed of the closed-loop poles will only lead to the system becoming dominated by the motor pole. This pole will move into the right-half plane as the PD gains are increased. These plots highlight the fact that the variable-pitch controller essentially cancels out the effect of the motor dynamics on the system. The same phenomenon occurs in the vertical-control case.

Fig. 10(c) shows the same reference commands but with a much more aggressive controller. The tracking performance is clearly better with the more aggressive controller, and this performance is only attainable with variable-pitch control as the motor pole goes unstable in the fixed-pitch case. Fig. 11 also shows the error improvement over the range of commanded roll rates using the nominal 

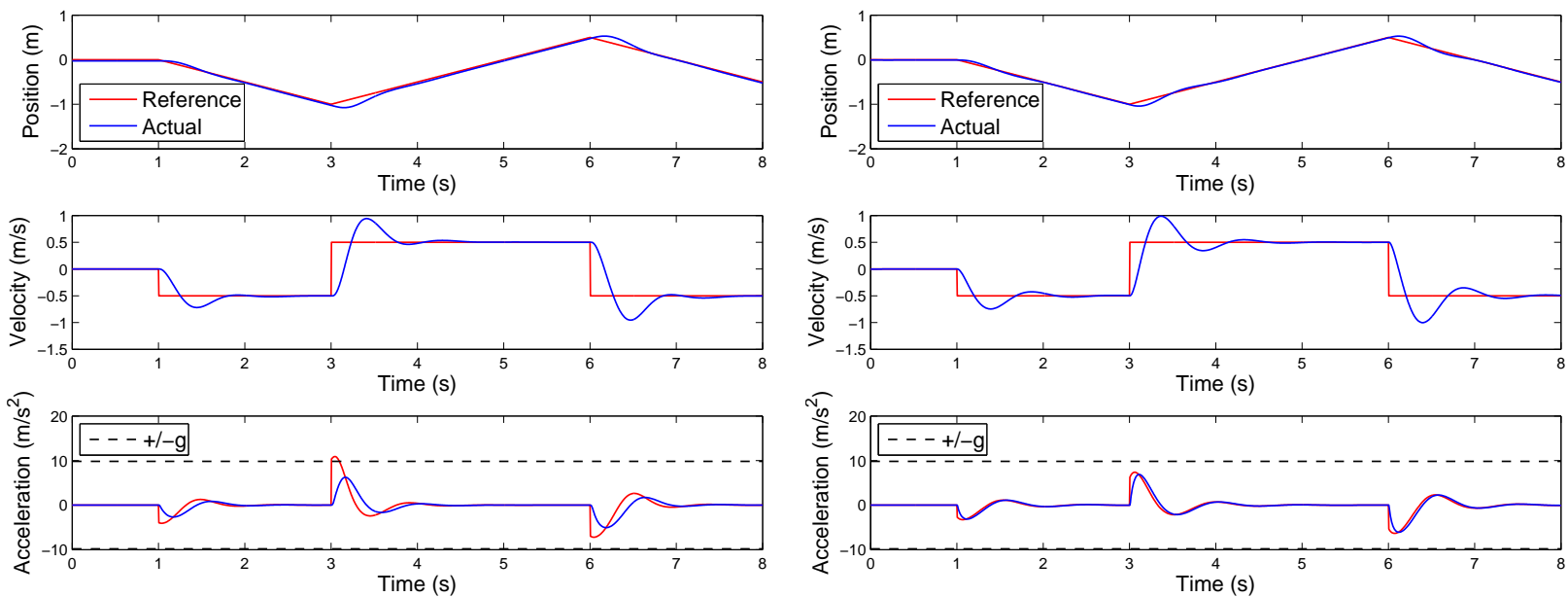

(a) Voltage Control

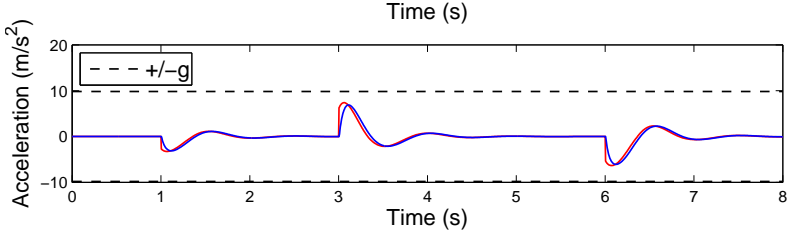

(b) Pitch Control

Figure 7. Vertical tracking at $0.5 \mathrm{~m} / \mathrm{s}$
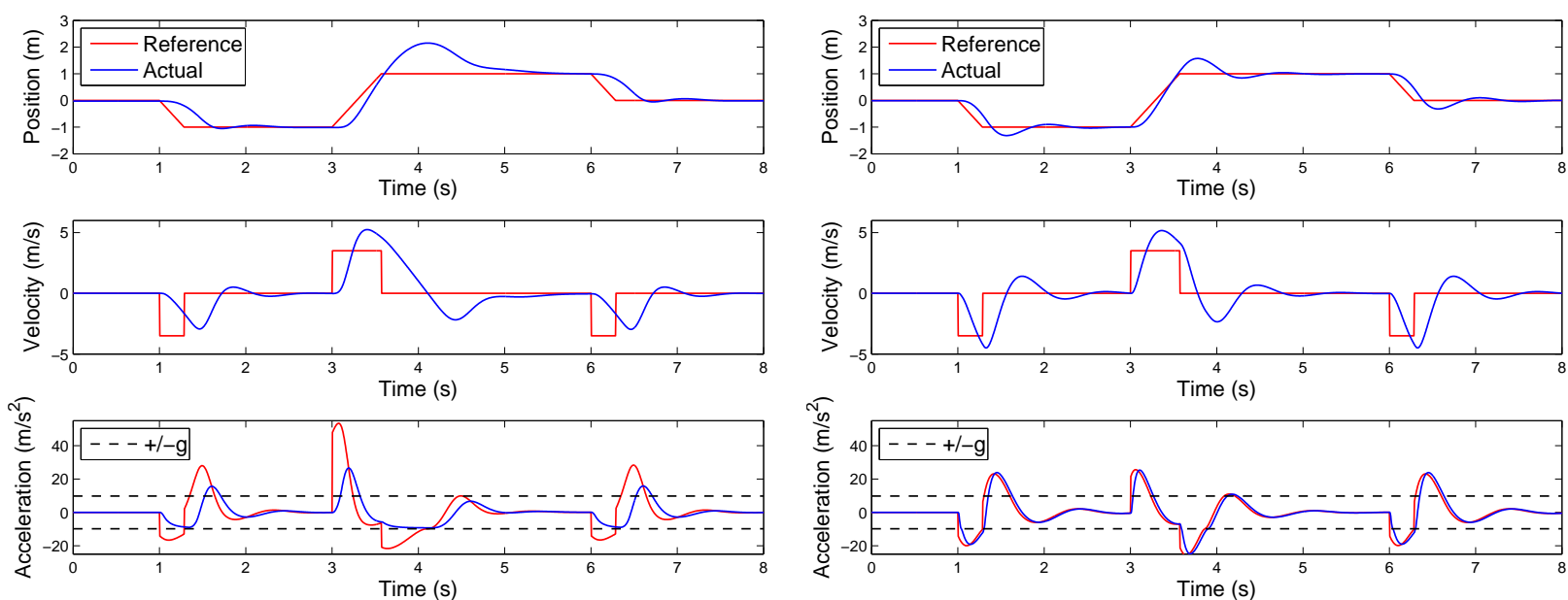

(a) Voltage Control
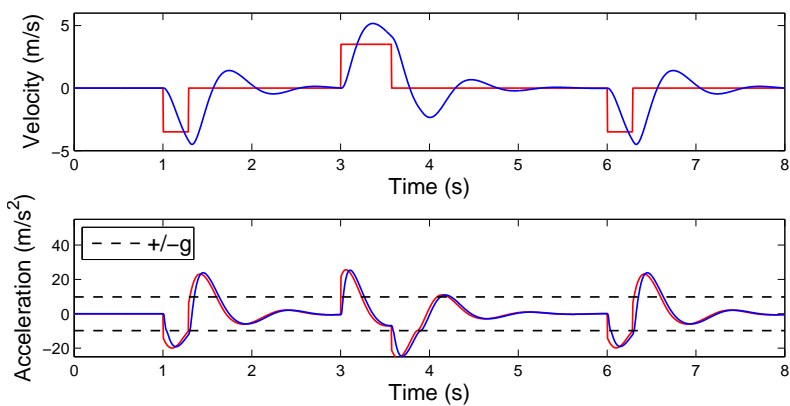

Figure 8. Vertical tracking at $3.5 \mathrm{~m} / \mathrm{s}$
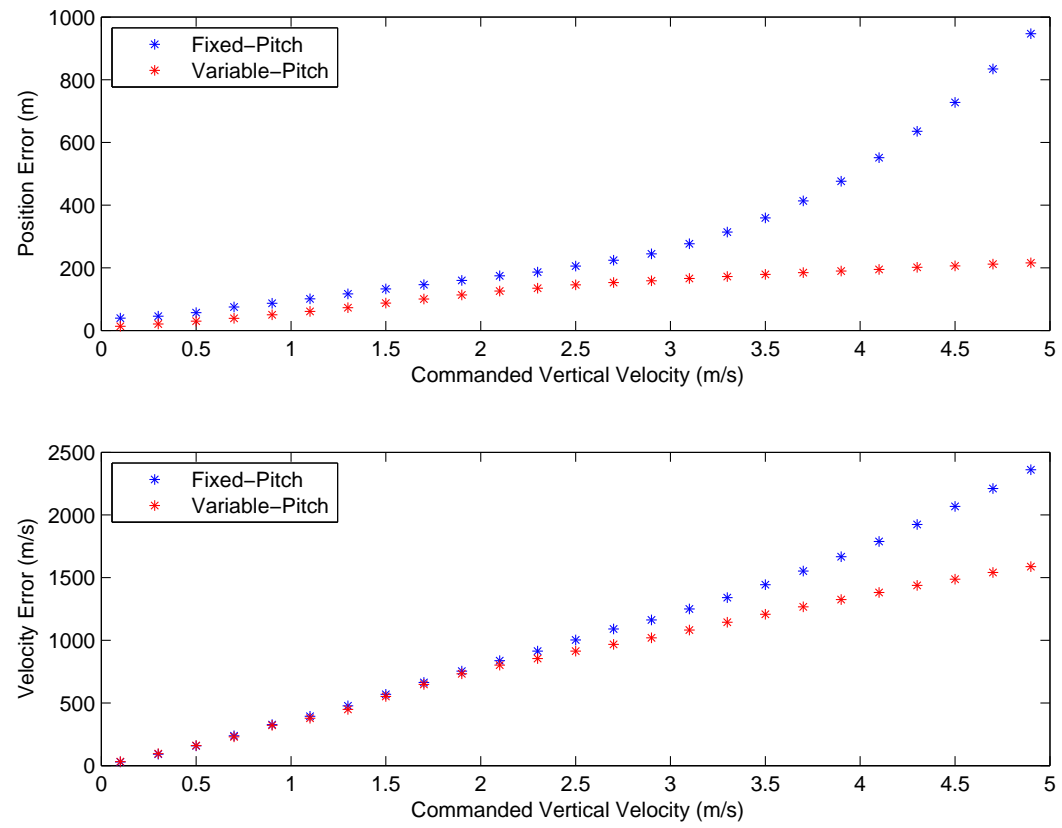

Figure 9. Vertical tracking error

11 of 17

American Institute of Aeronautics and Astronautics 

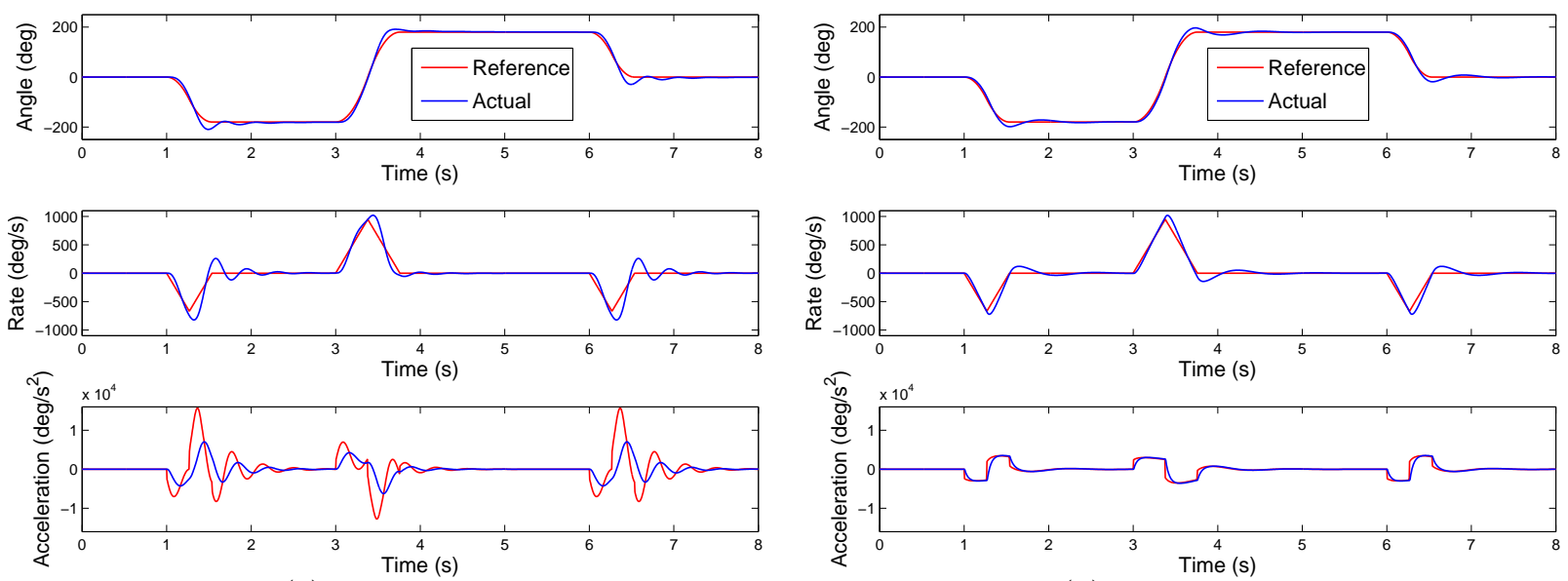

(a) Fixed-Pitch

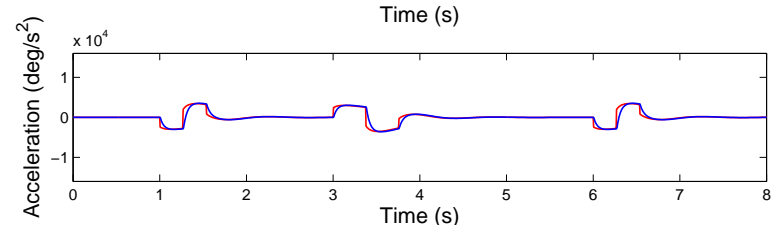

(b) Variable-Pitch
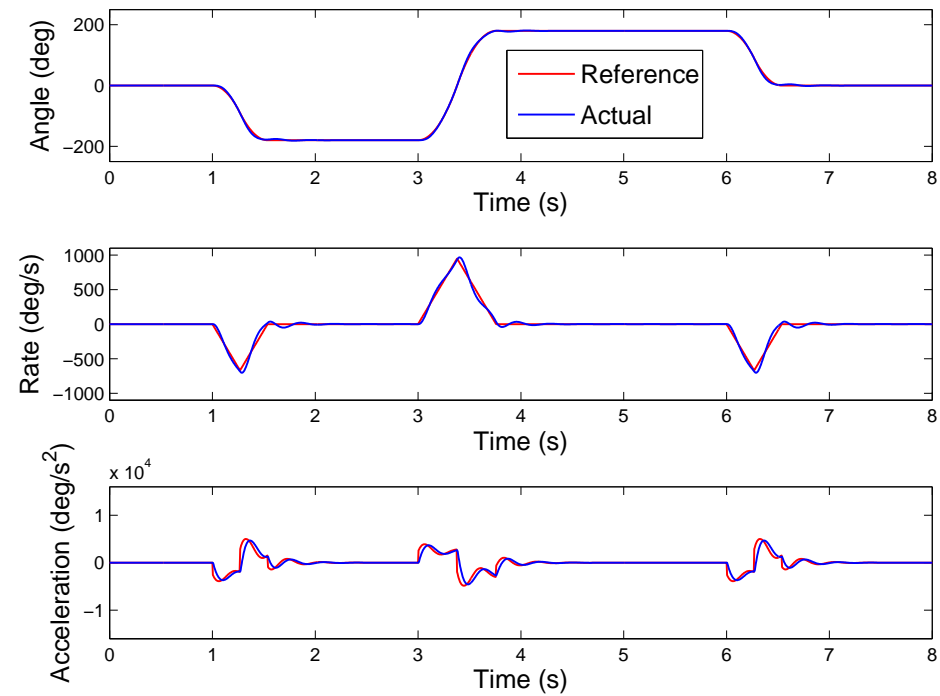

(c) Aggressive Variable-Pitch

Figure 10. Roll tracking at $2500 \mathrm{deg} / \mathrm{s}^{2}$
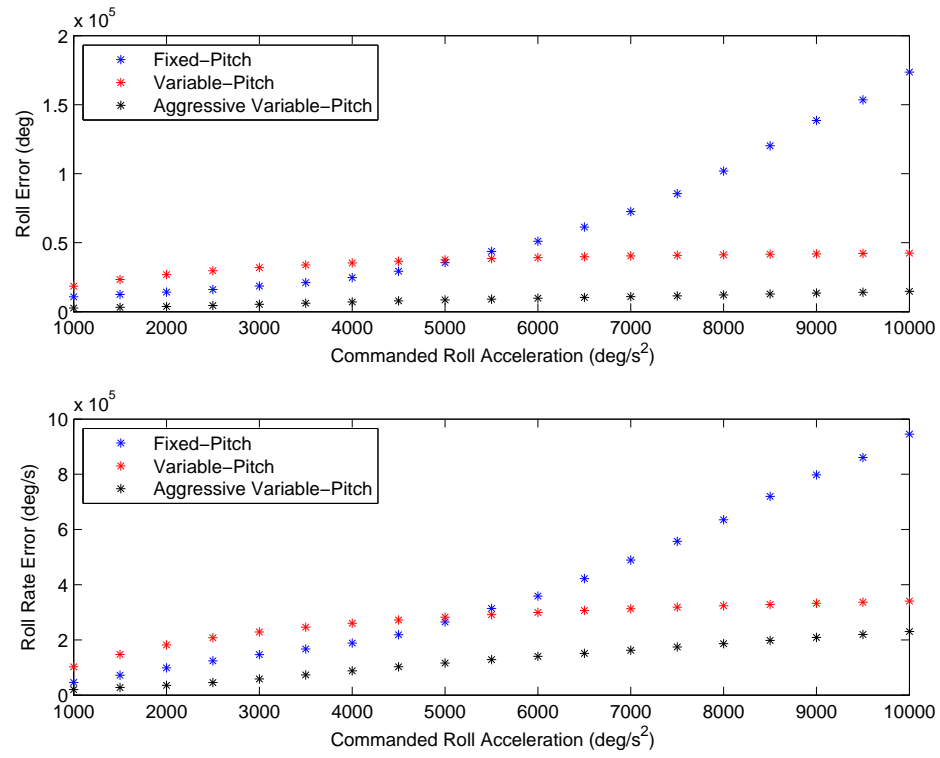

Figure 11. Roll tracking error 


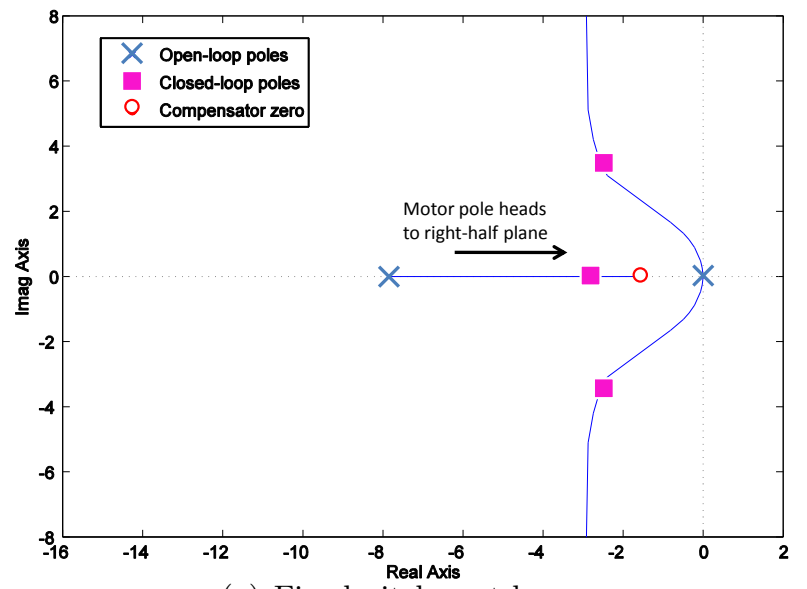

(a) Fixed-pitch root locus

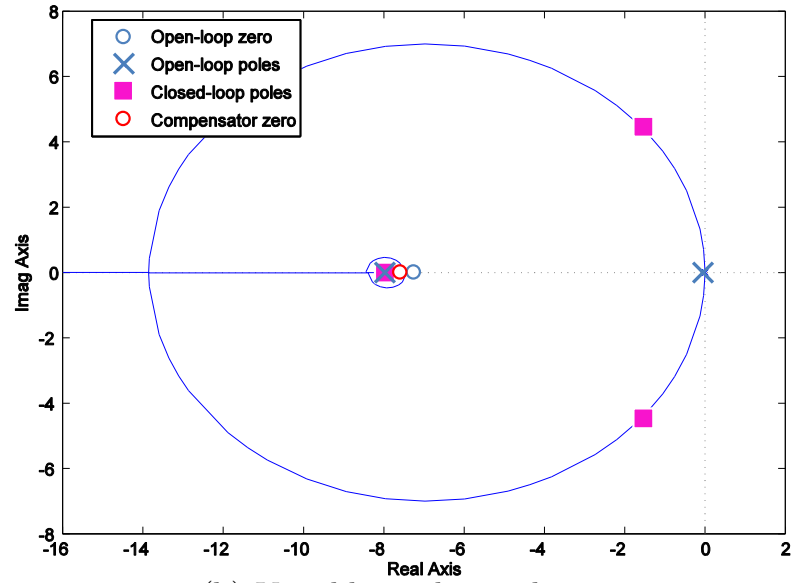

(b) Variable-pitch root locus

Figure 12. Root locus for fixed-pitch and variable-pitch propellers with a PD controller. In the fixed-pitch controller, the compensator zero is moved to the right to pull the poles at the origin to the left. In doing so, the motor pole heads to the right, eventually dominating the response and potentially causing the system to go unstable. The direct feed through term in the variable-pitch actuator, however, adds a zero near the motor pole allowing the poles at the origin to be pulled far into the left half plane.

and more aggressive controllers. The variable-pitch controller can be tuned to deliver much more aggressive responses than the fixed-pitch controller can.

\section{Experimental Testing}

The simulation data from Section IV is validated in this section with experimental testing of the quadrotor while it is attached to a test fixture that restricts motion to a single axis as shown in Fig. 13. The quadrotor is commanded to follow a series of roll and roll rate commands similar to those given in the rotational simulation above. Several different trajectory profiles are tracked with varying commanded accelerations. The same control strategy (PD) that was used in simulation is used in the experiments.

Fig. 14 shows experimental results of the quadrotor tracking an angular acceleration reference command of $2500 \mathrm{deg} / \mathrm{s}^{2}$. As predicted from the simulation, the angle and angular rate tracking for both the fixed-pitch and variable-pitch cases are similar. The vehicle's inertia is small enough that despite being a slower actuator, the fixed-pitch case can track the reference input well.

The difference between the two actuators is apparent, however, in the commanded values shown in Figures 14(c) and 14(d). These plots show the commands generated by the on-board controller and sent to the actuators. Both the commands to the motor via the ESC and the commands to the pitch via the servo are values between 0 and 250. The dashed green line in each plot shows the upper saturation limit of the actuators. Note that in the fixed-pitch case, the motor commands are nearly saturated during two of the flips while in the variable-pitch case, the servo and motor commands remain well within the saturation bounds.

The saturation issues are even more apparent as the commanded acceleration is increased. Fig. 15 shows similar plots but where the commanded acceleration is $5000 \mathrm{deg} / \mathrm{s}^{2}$. Fig. 15(c) shows that the motor commands in the fixed-pitch case are saturated for the majority of the first and third flips. The variable-pitch case performs the same maneuvers with less error while only briefly saturating the pitch command. The plots of the commanded values emphasize the ability of the variable-pitch actuator to quickly track reference commands.

For each of the experimental data sets, a measure of the tracking error is computed by summing 


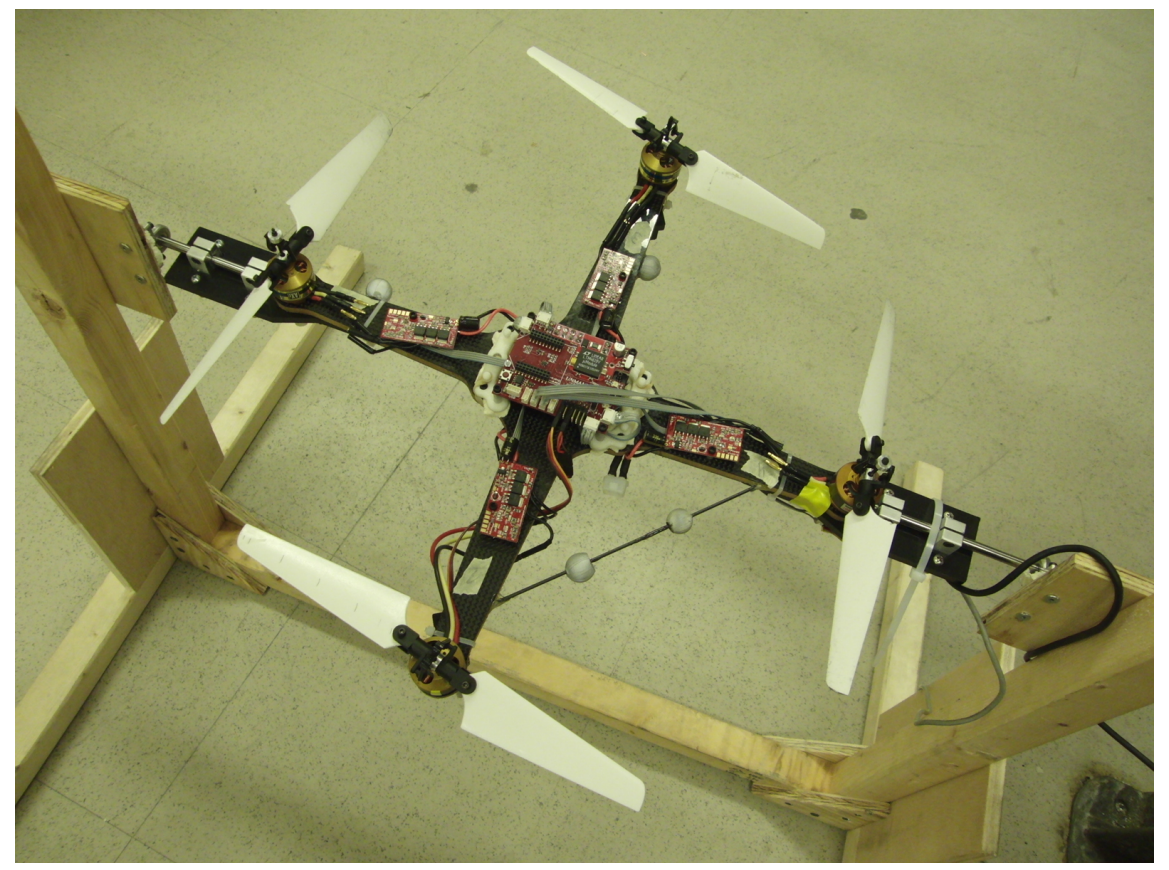

Figure 13. Quadrotor attached to roll test stand. Bearings mounted to the wooden frame allow for rotations about a single axis. The test stand is used for testing aggressive roll commands.

the absolute value of the difference between the reference command and the actual value over the entire data set. These error values are shown in Fig. 16. For a commanded acceleration of $10,000 \mathrm{deg} / \mathrm{s}^{2}$ the quadrotor reaches a roll rate of $1400 \mathrm{deg} / \mathrm{s}$, well into the agile flight regime. As predicted by simulation, the quadrotor performs better with the variable-pitch propellers than with fixed-pitch propellers.

\section{Conclusions}

This papers presents the design, analysis and experimental testing of a variable-pitch quadrotor. Section II shows that variable pitch actuation comes at the cost of adding a relatively small amount of complexity and weight to existing fixed-pitch designs. With this cost, however, comes the benefit of a fundamental increase in performance and capability as shown in Section III. The ability to control thrust using motor voltage in addition to propeller pitch allows for a significantly faster rate of change in thrust as well as the ability to choose between more-efficient or more-agile operating regimes.

The simulation and experimental results in Sections IV and V show that in nominal (non-agile) flight maneuvers there is little difference between fixed- and variable-pitch actuation. However, in more aggressive maneuvers it is apparent that fixed-pitch actuation suffers from control saturation well before the variable-pitch configuration. The fixed-pitch saturation leads to significantly larger errors in attitude rate and position tracking for large commanded angular and linear accelerations, respectively. Thus variable-pitch actuation increases the potential for agile maneuvering by expanding the control saturation limits.

Finally, the variable pitch quadrotor has the fundamental advantage of being able to reverse thrust very quickly. Not only does this result in more accurate attitude and acceleration command tracking, but also expands the envelope of attainable agile maneuvers. Aerobatic maneuvers such as the "tic-toc" and "split-S" that have been demonstrated by conventional helicopters are extremely 


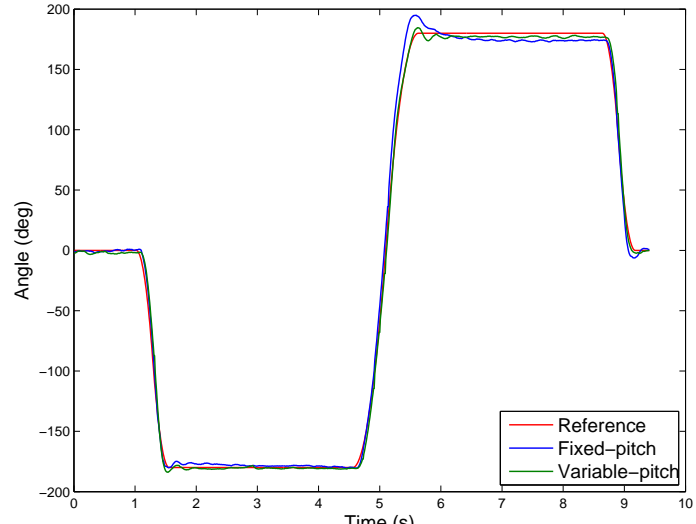

(a) Roll tracking
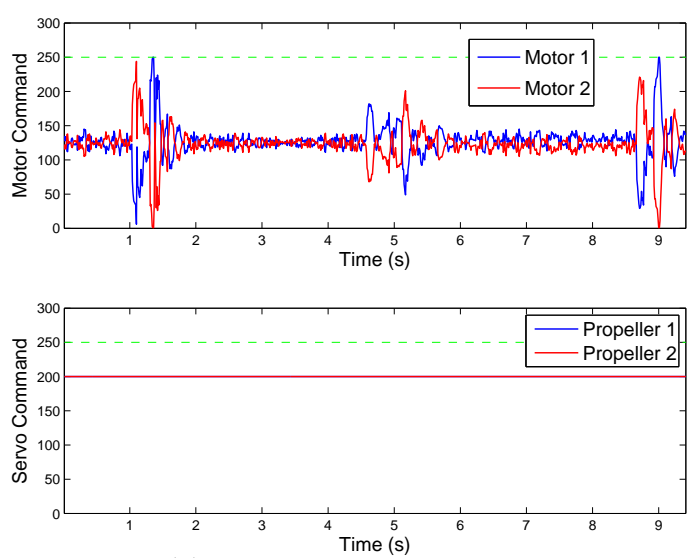

(c) Fixed-pitch commands

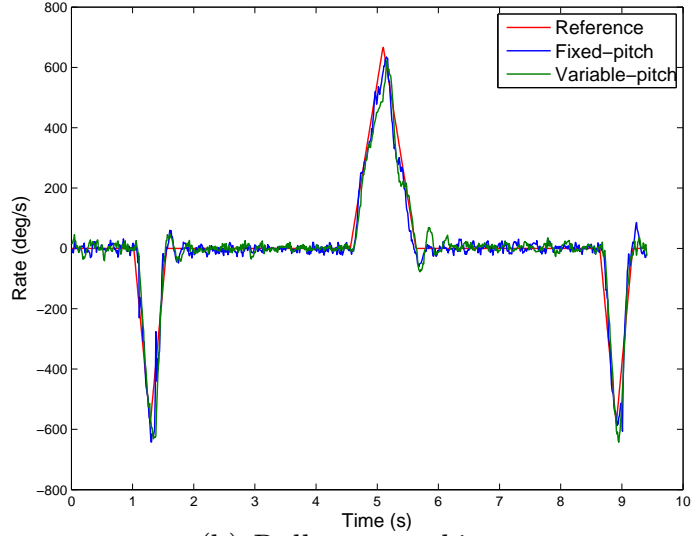

(b) Roll rate tracking
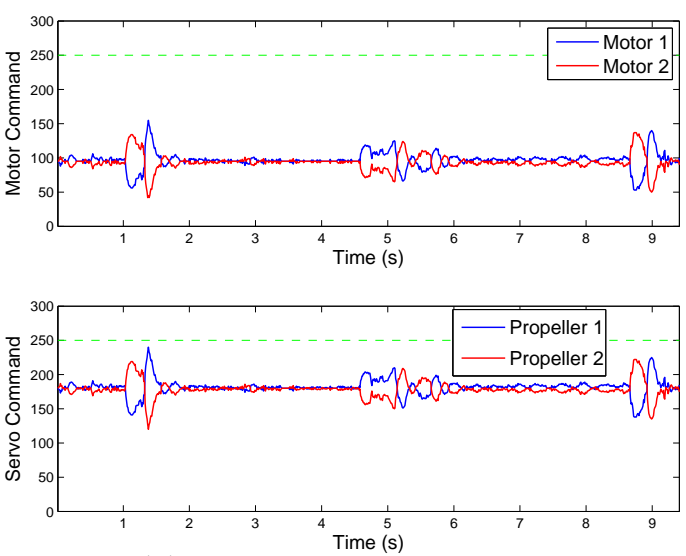

(d) Variable-pitch commands

Figure 14. Experimental roll tracking at $2500 \mathrm{deg} / \mathrm{s}^{2}$

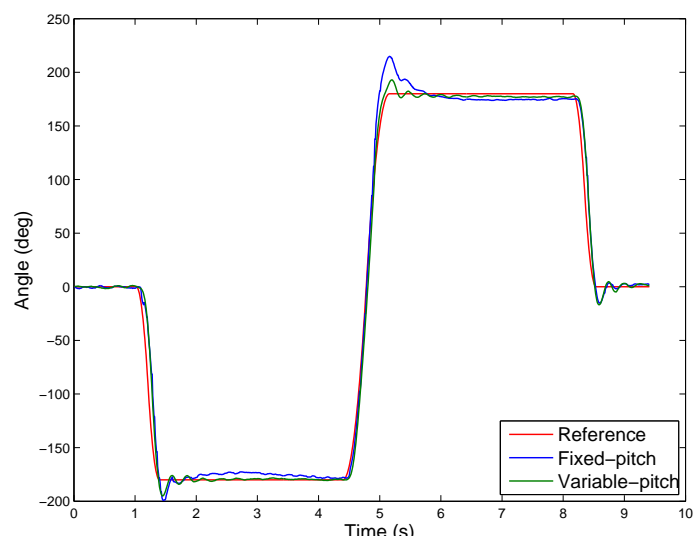

(a) Roll tracking
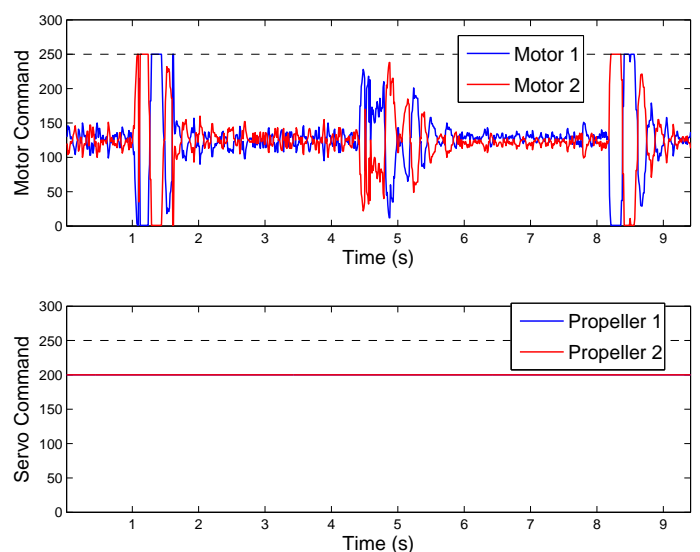

(c) Fixed-pitch commands

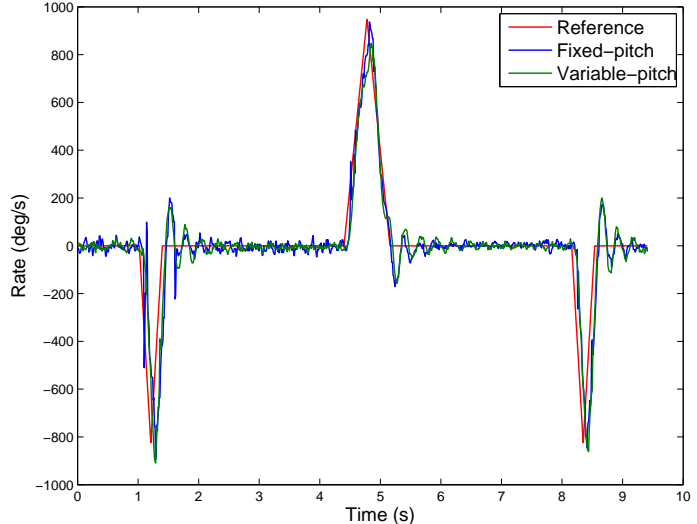

(b) Roll rate tracking
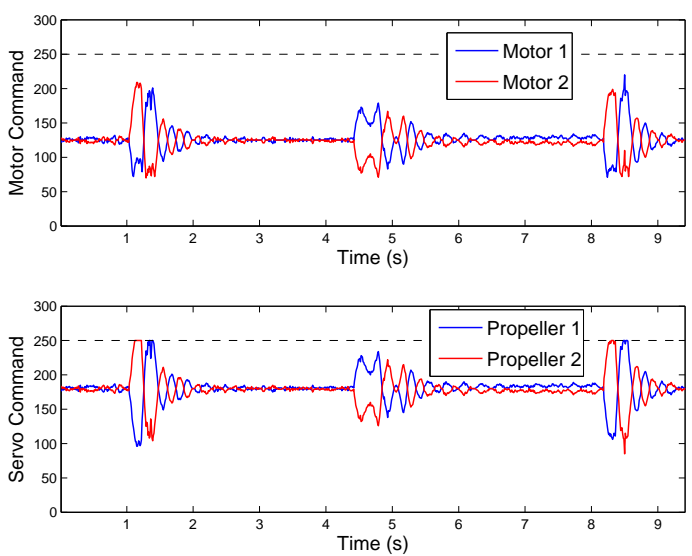

(d) Variable-pitch commands

Figure 15. Experimental roll tracking at $5000 \mathrm{deg} / \mathrm{s}^{2}$

15 of 17

American Institute of Aeronautics and Astronautics 

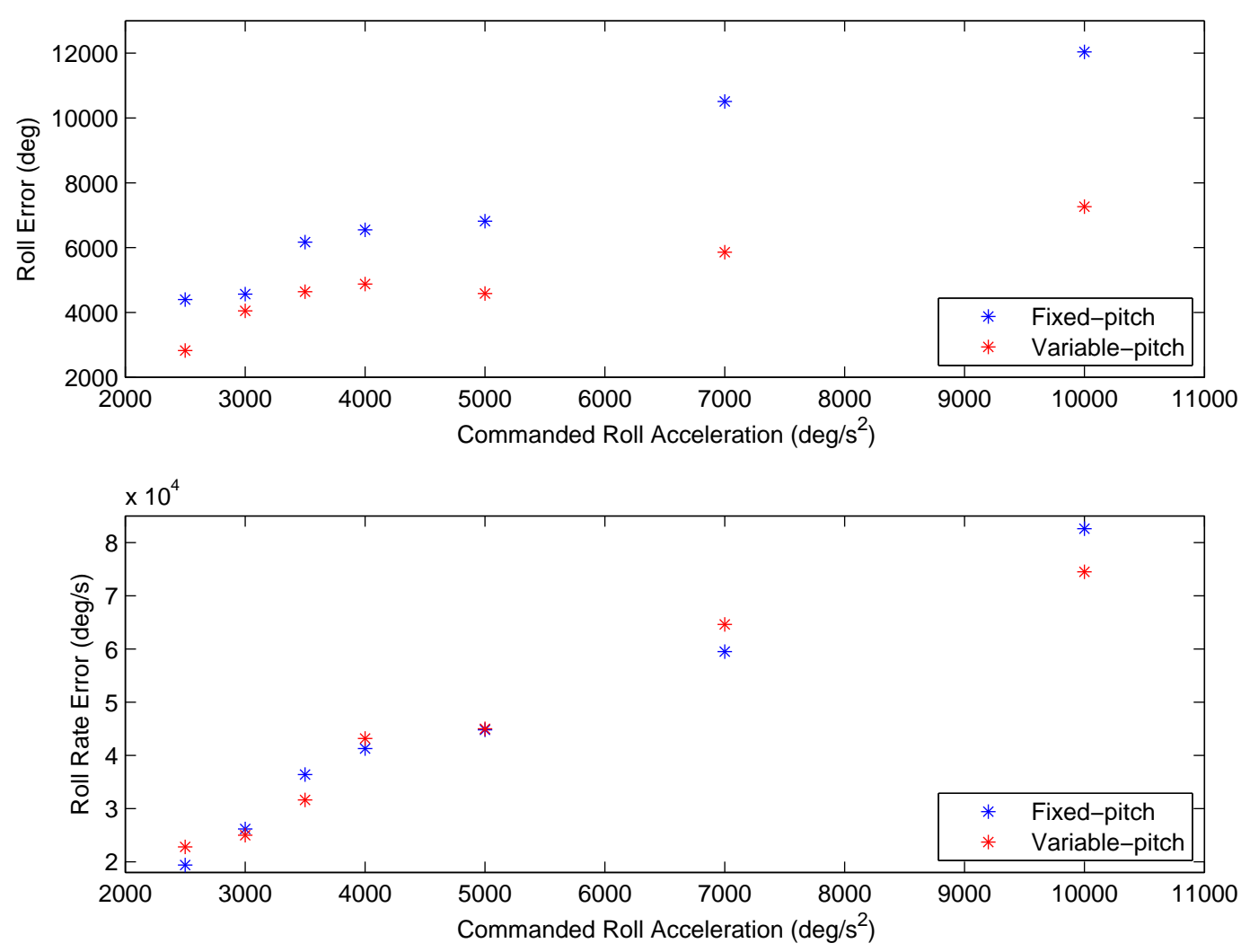

Figure 16. Experimental roll and roll-rate tracking error as a function of increasing commanded roll acceleration.

difficult for traditional quadrotors, but might be obtainable with variable-pitch actuation.

Future work will continue to experimentally demonstrate agile and aggressive maneuvering with the variable-pitch quadrotor hardware, and compare the performance with that of a fixedpitch counterpart. Further development of the control allocation strategy will also be required in order to choose the best combination of voltage and pitch control through the various nominal and agile flight envelopes.

\section{Acknowledgments}

This paper is based upon work supported by Aurora Flight Sciences and the National Science Foundation Graduate Research Fellowship under Grant No. 0645960. The authors also acknowledge the support of Boeing Research \& Technology.

\section{References}

${ }^{1}$ Amir, M. and Abbass, V., "Modeling of Quadrotor Helicopter Dynamics," International Conference on Smart Manufacturing Application (ICSMA 2008), April 2008, pp. 100 -105.

${ }^{2}$ Erginer, B. and Altug, E., "Modeling and PD Control of a Quadrotor VTOL Vehicle," IEEE Intelligent Vehicles Symposium, June 2007, pp. $894-899$.

${ }^{3}$ Alpen, M., Frick, K., and Horn, J., "Nonlinear modeling and position control of an industrial quadrotor with on-board attitude control," IEEE International Conference on Control and Automation, dec. 2009, pp. 2329 -2334.

${ }^{4}$ Kim, J., Kang, M., and Park, S., "Accurate Modeling and Robust Hovering Control for a Quad-rotor VTOL Aircraft," Journal of Intelligent and Robotic Systems, Vol. 57, No. 1, 2010, pp. 9-26.

${ }^{5}$ Huang, H., Hoffmann, G., Waslander, S., and Tomlin, C., "Aerodynamics and control of autonomous quadrotor 
helicopters in aggressive maneuvering," IEEE International Conference on Robotics and Automation (ICRA), May 2009, pp. $3277-3282$.

${ }^{6}$ Lupashin, S., Schollig, A., Sherback, M., and D'Andrea, R., "A simple learning strategy for high-speed quadrocopter multi-flips," IEEE International Conference on Robotics and Automation (ICRA), IEEE, 2010, pp. 1642-1648.

${ }^{7}$ Gillula, J., Huang, H., Vitus, M., and Tomlin, C., "Design of guaranteed safe maneuvers using reachable sets: Autonomous quadrotor aerobatics in theory and practice," IEEE International Conference on Robotics and Automation (ICRA), 2010, pp. 1649-1654.

${ }^{8}$ Michael, N., Mellinger, D., Lindsey, Q., and Kumar, V., "The GRASP Multiple Micro-UAV Testbed," IEEE Robotics $\&$ Automation Magazine, Vol. 17, No. 3, 2010, pp. 56-65.

${ }^{9}$ Pounds, P. and Mahony, R., "Design principles of large quadrotors for practical applications," IEEE International Conference on Robotics and Automation (ICRA), 2009, pp. 3265-3270.

${ }^{10}$ Mellinger, D., Michael, N., and Kumar, V., "Trajectory generation and control for precise aggressive maneuvers with quadrotors," Int. Symposium on Experimental Robotics, 2010.

${ }^{11}$ Mellinger, D. and Kumar, V., "Minimum Snap Trajectory Generation and Control for Quadrotors," IEEE International Conference on Robotics and Automation (ICRA), 2011.

${ }^{12}$ Gavrilets, V., Frazzoli, E., Mettler, B., Piedmonte, M., and Feron, E., "Aggressive Maneuvering of Small Helicopters: A Human Centered Approach," International Journal of Robotics Research, Vol. 20, October 2001, pp. $705-807$.

${ }^{13}$ Abbeel, P., Coates, A., Quigley, M., and Ng, A. Y., "An application of reinforcement learning to aerobatic helicopter flight," In Advances in Neural Information Processing Systems 19, MIT Press, 2007, p. 2007.

${ }^{14}$ d'Ambrosio, G. and Navoni, R., "HG3 Willy [Video]," accessed July, 18, 2011 (http://youtu.be/ M4uXmekZk-4).

${ }^{15}$ Borenstein, J., "The Hoverbot, An Electrically Powered Flying Robot," unpublished paper, 1992, (http: //www.cs.cmu.edu/ motionplanning/papers/sbp_papers/integrated1/borenstein_hovercraft.pdf).

${ }^{16}$ Chen, H., "Variable-Pitch Quadrotor [Video]," accessed July, 18, 2011 (http://youtu.be/fkSx3fSz0tE).

${ }^{17}$ Michini, B., Redding, J., Ure, N. K., Cutler, M., and How, J. P., "Design and Flight Testing of an Autonomous Variable-Pitch Quadrotor," IEEE International Conference on Robotics and Automation (ICRA), IEEE, 2011.

18 "Unmanned Innovation Inc," 7 Sunset Way, Suite 100, Henderson, NV 89014 info@unmannedinnovation. com.

${ }^{19}$ Drela, M., "XFOIL- An analysis and design system for low Reynolds number airfoils," Low Reynolds Number Aerodynamics, 1989, pp. 1-12, (http://web.mit.edu/drela/Public/web/xfoil/).

${ }^{20}$ Bresciani, T., Modelling, identification and control of a quadrotor helicopter, Department of Automatic Control, Lund University, 2008.

${ }^{21}$ Drela, M., "QPROP Users Guide," 2009, (http://web.mit.edu/drela/Public/web/qprop/).

${ }^{22}$ Bristeau, P., Martin, P., Salaun, E., and Petit, N., "The role of propeller aerodynamics in the model of a quadrotor UAV," European Control Conference, 2009.

${ }^{23}$ Franklin, G., Powell, J., and Emami-Naeini, S., Feedback Control of Dynamic Systems, Prentice Hall, 2006. 